\title{
K-SHELL FLUORESCENCE YIELD OF THORIUM PRESENT IN ITS COMPOUND
}

\author{
L. D. Horakeri*, S. G. Bubbly** and \\ S. B. Gudennavar*** $†$
}

\begin{abstract}
A simple method, proposed by us earlier, - $2 \pi$ geometrical configuration coupled with the thickness criterion - to measure the Kshell $X$-ray fluorescence parameters is extended to high $Z$ materialThorium. The fluorescent $X$-rays are generated by a weak $C_{0}-57$ radioactive source and detected by employing a $\mathrm{Nal}(\mathrm{TI})$ detector spectrometer. Measured value is compared with the best fitted value produced by Hubbell et al. and with the experimental value of Balakrishno et al. We found good agreement with each other, thus establishing the applicability of our simple method for measuring fluorescence yield of high Z element.
\end{abstract}

Keywords: $K X$-ray, Fluorescence Yield and $2 \pi$ geometrical configuration

- Department of Physics, S. K. Ants College and H. S. Kotambri Science Institute, Vidyanagar, Hubli - 580031 , Karnataka, India.

** Post Graduate Department of Physics, Christ University, Bangalore-560 029

***† E-mail: shivappa.b.gudennavar@christuniversity.in 


\section{Introduction}

$X$-ray fluorescence (XRF) phenomena has been utilised in a wide variety of fields. This demands not only the accurate measurements of XRF parameters but also a wide variety of geometrical conditions to meet the requirements in diverse fields such as health physics, industry, forensic science, archaeology and so on. In recent years, we have developed, successfully, a simple and an elegant method to obtain XRF parameters accurately [1-5]. The crucial features of our method include the provision for large solid angle (almost $2 \pi$ sr.) subtended by the detector at the source as well as at the target. This helps to estimate accurately the number of photons incident on the target, which is an important quantity in the measurement of fluorescence parameters. In this geometrical configuration, the number of photons that enter into the detector, in the absence of target, is same as the number of photons that incident on the target.

The other feature is the estimation of number of fluorescent $X$-rays that are emitted from the target atoms in all directions. This is usually affected by the self-attenuation in the target, for the $X$-rays emitted in a particular portion of the target are attenuated in the subsequent layers on either side of that portion. Bambynek et al. [6], have suggested that the target thickness should be optimised in such a way that the production of $X$-rays must be predominant compared to their attenuation in the target itself (self-attenuation); this is necessary to obtain the values of XRF parameters accurately. Following their suggestion, we found that a target whose thickness lies between $0.75 \leq \beta \leq 0.95$, is most suitable for XRF parameters measurements; here $\beta$ is the self-attenuation factor whose expression is modified to suit our method and is given by

$$
\beta=\frac{1-\exp \left(-\left(\mu_{i}+\mu_{\mathrm{e}}\right) t\right)}{\left(\mu_{\mathrm{i}}+\mu_{\mathrm{e}}\right) t}
$$

which differs from the one that is normally used by others over the years. The details of these are discussed in our earlier papers [ $1-5]$. In this paper, we show that our simple method can be extended to cover high Z materials also and thus establishing the applicability of this method for material of any $Z$.

\section{Experimental}

Uniform targets, with thickness that satisfies the condition $0.75 \leq \beta \leq 0.95$, are prepared from the compounds of Thorium $(Z=90)$. In Table 1, we give the details 
of the target. Targets are excited by gamma radiations $(122 \mathrm{keV}(85 \%)$ and $136 \mathrm{keV}$ (11\%)) from a weak ${ }^{57} \mathrm{Co}$ radioactive source $\left(\sim 10^{4} \mathrm{~Bq}\right)$, which was procured as a standard gamma source from BRIT, Mumbai, India. The X-ray spectrometer, consisting of a $2^{\prime \prime} \times 1 \frac{3 / 4}{4}$ thick Nal(Tl) crystal coupled to MCA, was employed to detect and to measure the intensity of $X$-rays. The spectrometer was calibrated using several X-ray and gamma ray sources. Its linearity and stability were checked before its use.

Table 1. Calculated values of self-attenuation factor, $b$, for selected target thickness at excitation energy $123.6 \mathrm{keV}$.

\begin{tabular}{lccccc}
\hline Compound & $\begin{array}{c}\text { Atomic number } \\
Z\end{array}$ & $\begin{array}{c}\mu_{i} \\
\left(\mathrm{~cm}^{2} / \mathrm{g}\right)\end{array}$ & $\begin{array}{c}\mu_{\mathrm{e}} \\
\left(\mathrm{cm}^{2} / \mathrm{g}\right)\end{array}$ & $\begin{array}{c}\dagger \\
\left(\mathrm{g} / \mathrm{cm}^{2}\right)\end{array}$ & $\beta$ \\
\hline Thorium nitrate & 90 & 1.706 & 0.938 & 0.03342 & 0.957 \\
\hline
\end{tabular}

The experimental arrangement made in the present investigation is as shown in Fig. 1. The incident intensity (source intensity) and the transmitted intensity of photons plus the intensity of fluorescent $X$-rays of interested element are then measured without and target in place respectively. Then the number of photons incident on the target, and the number of fluorescent $X$-rays emitted in all forward angles are measured by making use of spectrum subtraction facility built-in in the MCA. The details of the procedure were discussed in our earlier papers [1-5].

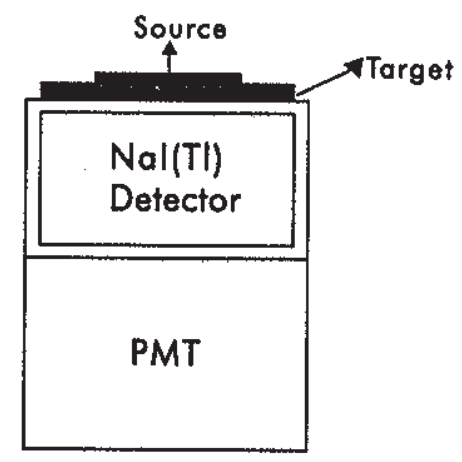

Figure 1. Schematic diagram of the experimental arrangement.

Since Nal(Ti) detector spectrometer is not capable of resolving the gamma rays of energies $122 \mathrm{keV}$ and $136 \mathrm{keV}$, we used the weighted average of these energies, $123.6 \mathrm{keV}$, for calculation purposes. 


\section{Results and Discussion}

The fluorescence yield, $\omega_{k}$ value of thorium is determined in several trials to check the reproducibility and reliability of our method. In Table 2, we give the $\omega_{k}$ values along with their associated errors obtained in several trials for thorium nitrate along with the weighted average of these $\omega_{k}$ values and the fitted values produced by Hubbell et al. [7] are also given.

Table 2. Measured values of fluorescence yield, $\omega_{k}$, for Thorium nitrate at excitation energy $123.6 \mathrm{keV}$ in six trials.

\begin{tabular}{cc}
\hline Trial No. & $\omega_{\mathrm{k}}$, for Thorium nitrate \\
\hline 1. & $0.975 \pm 0.014$ \\
2. & $0.980 \pm 0.014$ \\
3. & $0.988 \pm 0.014$ \\
4. & $0.969 \pm 0.014$ \\
5. & $0.965 \pm 0.014$ \\
6. & $0.979 \pm 0.014$ \\
Weighted average value & $0.976 \pm 0.006$ \\
Hubbell et al.'s value [7] & 0.9691 \\
\hline
\end{tabular}

From Table 2 , it is clear that $\omega_{k}$ values are not only in agreement amongst themselves but also in agreement with the weighted average values and the standard fitted values. Thus establishes the credibility of our method.

In Table 3, we compare our experimental values with others experimental values obtained by adopting different geometries and methods and we see that our experimental values are in good agreement with the others experimental values indicating that our simple method also yield $\omega_{k}$ values on par with those measured using elaborate arrangements. 
Table 3. Comparison of fluorescence yield, $\omega_{k}$, value of thorium nitrate with standard fitted values of Hubbell et al. and others experimental values.

\begin{tabular}{lcccc}
\hline Compound & $Z$ & $\begin{array}{c}\text { Present } \\
\text { experimental value }\end{array}$ & $\begin{array}{c}\text { Standard fitted } \\
\text { value [7] }\end{array}$ & $\begin{array}{c}\text { Others experimental } \\
\text { value [8] }\end{array}$ \\
\hline Thorium nitrate & 90 & $0.976 \pm 0.006$ & 0.9691 & $0.974 \pm 0.057$ \\
\hline
\end{tabular}

\section{Conclusion}

From the results discussed above, we conclude that this work combined with our earlier measurements of $\omega_{k}$ values for various materials of different $Z$, clearly establishes the applicability of our simple method for measurement of fluorescence yield for materials of any $Z$ but with suitable detector spectrometer that matches the $X$-ray energies.

\section{References}

1. Horakeri L. D., Hanumaiah B. and Thontadarya S. R. X-ray Spectrom. 1997; 26: 69-74.

2. Horakeri L. D., Hanumaiah B. and Thontadarya S. R. X-ray Spectrom. 1998; 27: 344-348.

3. Gudennavar S. B., Badiger N. M., Thontadarya S. R. and Hanumaiah B. Radiat. Phys, and Chem. 2003; 68: 721-726.

4. Gudennavar S. B., Badiger N. M., Thontadarya S. R. and Hanumaiah B. Radiat. Phys. and Chem. $2003 ; 68:$ 745-750.

5. Gudennavar S. B., Badiger N. M., Thontadarya S. R. and Hanumaiah B. Am. Jour. Phys. $2003 ; 71: 822-825$.

6. Bambynek W., Crasemann B., Fink R. W., Freund H. V., Mark H., Swift D. C., Price R. C. and Venugopal Roo Rev. Mod. Phys. 1972; 44: 716-813.

7. Hubbell J. H., Trehan P. N., Nirmal Singh, Chand B., Mehto D., Garg M. L., Surinder Singh and Puri S. Jour. Phys. Chem. Ref. Dota 1994; 23: 339.

8. Balakrishna K. M., Govind Nayak N., Lingappa N. and Siddappa K. Jour. Phys. B: Atom. Mol. Opt. Phys. 1994; 27: 715. 